\title{
Association of Aortic Compliance and Brachial Endothelial Function with Cerebral Small Vessel Disease in Type 2 Diabetes Mellitus Patients: Assessment with High-Resolution MRI
}

\author{
Yan Shan, ${ }^{1}$ Jiang Lin, ${ }^{1}$ Pengju Xu, ${ }^{1}$ Mengsu Zeng, ${ }^{1}$ Huandong Lin, ${ }^{2}$ and Hongmei Yan ${ }^{2}$ \\ ${ }^{1}$ Department of Radiology, Zhongshan Hospital, Fudan University and Shanghai Institute of Medical Imaging, Shanghai 200032, China \\ ${ }^{2}$ Department of Endocrinology, Zhongshan Hospital, Fudan University, Shanghai 200032, China \\ Correspondence should be addressed to Jiang Lin; lin.jiang@zs-hospital.sh.cn
}

Received 18 March 2016; Accepted 26 June 2016

Academic Editor: János Nemcsik

Copyright (c) 2016 Yan Shan et al. This is an open access article distributed under the Creative Commons Attribution License, which permits unrestricted use, distribution, and reproduction in any medium, provided the original work is properly cited.

\begin{abstract}
Objective. To assess the possible association of aortic compliance and brachial endothelial function with cerebral small vessel disease in type 2 diabetes mellitus (DM2) patients by using 3.0 Thigh-resolution magnetic resonance imaging. Methods. Sixty-two clinically confirmed DM2 patients ( 25 women and 37 men; mean age: $56.8 \pm 7.5$ years) were prospectively enrolled for noninvasive MR examinations of the aorta, brachial artery, and brain. Aortic arch pulse wave velocity (PWV), flow-mediated dilation (FMD) of brachial artery, lacunar brain infarcts, and periventricular and deep white matter hyperintensities (WMHs) were assessed. Pearson and Spearman correlation analysis were performed to analyze the association between PWV and FMD with clinical data and biochemical test results. Univariable logistic regression analyses were used to analyze the association between PWV and FMD with cerebral small vessel disease. Multiple logistic regression analyses were used to find out the independent predictive factors of cerebral small vessel disease. Results. Mean PWV was $6.73 \pm 2.00 \mathrm{~m} / \mathrm{s}$ and FMD was $16.67 \pm 9.11 \%$. After adjustment for compounding factors, PWV was found significantly associated with lacunar brain infarcts (OR $=2.00 ; 95 \%$ CI: 1.14-3.2; $P<0.05)$ and FMD was significantly associated with periventricular WMHs (OR = 0.82; 95\% CI: 0.71-0.95; $P<0.05)$. Conclusions. Quantitative evaluation of aortic compliance and endothelial function by using high-resolution MRI may be potentially useful to stratify DM2 patients with risk of cerebral small vessel disease.
\end{abstract}

\section{Introduction}

Type 2 diabetes mellitus (DM2) can cause many cardiovascular complications including ischemic stroke which is a leading cause of death $[1,2]$. Arterial endothelial dysfunction and arterial compliance abnormalities are early arterial changes in DM2 patients which occur earlier than structural abnormalities of vessel walls and clinical onset of cardiovascular complications [3-5]. Studies have shown that arterial stiffening is a strong predictor of future cardiovascular events and all-cause mortality and an independent predictor of fatal stroke $[6,7]$.

Cerebral small vessel disease is one of the common cardiovascular complications in DM2 patients [8]. There is report that the stroke risk in patients with DM2 is two to five times higher than that in patients with normal glucose [9]. If an association of arterial endothelial dysfunction and arterial compliance abnormalities with cerebral small vessel disease could be established, this would support the significance of measuring arterial endothelial dysfunction and arterial compliance abnormalities for early prediction, stratification, and prevention of this cardiovascular complication in DM2 patients. It has been reported that PWV is independently associated with cerebral small vessel disease in patients with type $1 \mathrm{DM}$ [10]. Another study has shown that FMD is associated with poor prognosis in patients with ischemic stroke [11]. Compared with DM1, DM2 has higher incidence; furthermore, its prevalence is increasing rapidly not only in developed countries but also in developing countries [12]. To our knowledge, there has been no report so far about association between aortic compliance and brachial endothelial function with cerebral small vessel disease in DM2 patients by using magnetic resonance imaging (MRI). 
The advantages of MRI include noninvasiveness, being without radiation, high soft tissue resolution, a large field of view, and being less operator-dependent. The previous study has demonstrated that high-resolution MRI can provide superior image quality and reproducibility for the assessment of arterial compliance and endothelial function of the central and peripheral arteries during a single examination [13]. In addition, MRI is superior in the evaluation of lacunar brain infarcts and white matter injuries resulted from cerebral small vessel disease [14].

Therefore, we attempt to assess the possible association between aortic compliance and endothelial function with cerebral small vessel disease in DM2 patients by using 3.0 T high-resolution MRI.

\section{Materials and Methods}

2.1. Study Population. We enrolled all patients with a diagnosis of DM2 at our diabetes and hypertension outpatient clinic between January 2010 and October 2012. A total of 62 DM2 patients (37 men, 25 women; mean age $56.8 \pm$ 7.5 years) were enrolled in our study. DM2 was defined as repeatedly measured fasting blood glucose $\geq 7.0 \mathrm{mmol} / \mathrm{L}$ or nonfasting glucose $\geq 11.1 \mathrm{mmol} / \mathrm{L}$ and the test results of glutamic acid decarboxylase antibody (GADA) were negative according to WHO criteria [15]. Diabetes duration was defined as the time from diagnosis of DM2 to MRI scan. All diabetic patients were on treatment with insulin alone or with combined insulin and other antidiabetic drugs. Blood pressure was measured at the time of MRI using a semiautomated sphygmomanometer. Pulse pressure was defined as follows: systolic blood pressure minus diastolic blood pressure. Patient blood was drawn for biochemical tests in the morning after an overnight fast within 2 weeks before MRI. Hypertension (i.e., systolic blood pressure > $140 \mathrm{mmHg}$ or diastolic blood pressure $>90 \mathrm{mmHg}$ or the use of antihypertensive medication [16]), body mass index (i.e., the patient's body weight in kilograms during MR imaging divided by the square root of the height of the patient in centimeters), smoking status (nonsmoker or smoker), and lipid profiles (total cholesterol, high-density lipoprotein, lowdensity lipoprotein, and triglycerides) were determined. This study was approved by the local medical ethics committee and all subjects gave informed consent to participate in the study.

2.2. MRI Protocol. Aortic and brachial artery imaging were performed using a 3.0 T MRI (Signa HDX, GE Medical Systems, Milwaukee, Wis, USA) with ECG-gated technique and an 8-channel phased array body coil. All brain examinations were also performed after arterial imaging using the same MR equipment with an 8-channel phased array head coil. Total imaging time was 45 minutes.

2.2.1. Aortic Arch PWV and Brachial FMD. Aortic arch PWV and brachial FMD were measured using a previously described protocol [12, 13]. Examination parameters are included in Table 1.

Figure 1 demonstrates assessment of aortic arch PWV between the ascending and the proximal descending aorta.
TABLE 1: MR imaging parameters for assessment of aortic arch PWV and FMD.

\begin{tabular}{lcc}
\hline Parameter & Aortic arch PWV & FMD \\
\hline Repetition time (TR) & $40.0 \mathrm{~ms}$ & $3.3 \sim 150 \mathrm{~ms}$ \\
Echo time (TE) & $5.0 \mathrm{~ms}$ & $1.5 \sim 1.8 \mathrm{~ms}$ \\
Slice thickness & $4.0 \mathrm{~mm}$ & $6.0 \mathrm{~mm}$ \\
Matrix size & $256 \times 192$ & $224 \times 224$ \\
Image resolution & $1.37 \mathrm{~mm} \times 1.37 \mathrm{~mm}$ & $1.16 \mathrm{~mm} \times 1.16 \mathrm{~mm}$ \\
Temporal resolution & $4.7-7.8 \mathrm{~ms}$ & $18.75 \sim 31.25 \mathrm{~ms}$ \\
Encoding velocity & $150 \mathrm{~cm} / \mathrm{s}$ & $/$ \\
\hline
\end{tabular}

An ECG-gated, spoiled gradient echo sequence with velocityencoding gradient for phase contrast was applied during breath-hold to the ascending and descending aorta at the level of the right pulmonary artery. Flow waveforms were obtained from the two cross-section planes by using the ReportCARD software on the workstation (GE AW 4.3). The distance (L) measurement was done manually by a series of short straight connected lines along the aortic luminal midline across the aortic arch at right pulmonary artery plane and PWV was calculated by dividing the distance around the arch of the aorta by the transit time between the arrivals of the systolic wave front at the two sites.

Figure 2 demonstrates assessment of FMD. The FIESTA sequence was used. The GE AW 4.3 workstation was used to measure the end diastolic artery cross-section area and to calculate the area change before and after compression. FMD was calculated through the following equation: diastolic function $=($ area after compression - baseline area before compression)/baseline area before compression $x$ $100 \%$. Maximum percentage change in brachial artery crosssectional area at end diastole was used to determine the response to the stimulus of cuff compression.

2.2.2. Evaluation of Cerebral Lesions with MRI. For evaluation of cerebral small vessel disease, a spin-echo T2-weighted imaging (T2WI), a fluid-attenuated inversion recovery (FLAIR) imaging, and a T1-weighted gradient echo imaging were performed. Acquisition parameters for T2WI were as follows: TR/TE, $3400 / 110 \mathrm{~ms}$, flip angle, $90^{\circ}$, field of view: $240 \mathrm{~mm}$, section thickness: $5 \mathrm{~mm}$, gap thickness: $1.5 \mathrm{~mm}$, and 21 sections. Acquisition parameters for the FLAIR were as follows: TR/TE/inversion time (IR): 9000/150/2250, flip angle: $90^{\circ}$, field of view: $240 \mathrm{~mm}$, section thickness: $5 \mathrm{~mm}$, gap thickness: $1.5 \mathrm{~mm}$, and 21 sections. Acquisition parameters for T1WI were as follows: TR/TE, 1750/150, flip angle, $90^{\circ}$, field of view: $240 \mathrm{~mm}$, section thickness: $5 \mathrm{~mm}$, gap thickness: $1.5 \mathrm{~mm}$, and 21 sections.

For cerebral small vessel disease, two entities including lacunar brain infarcts and white matter hyperintensities (WMHs) were evaluated. Lacunar brain infarcts (Figure 3) were defined as small (but $>3 \mathrm{~mm}$ in size) cavities within the brain parenchyma, with similar signal intensity to that of cerebrospinal fluid on all pulse sequences, surrounded by an area of high signal intensity on T2 and FLAIR images $[17,18]$. Their presence was defined on a binary scale: absent (0) or present (1). WMHs (Figure 4) were defined as areas 


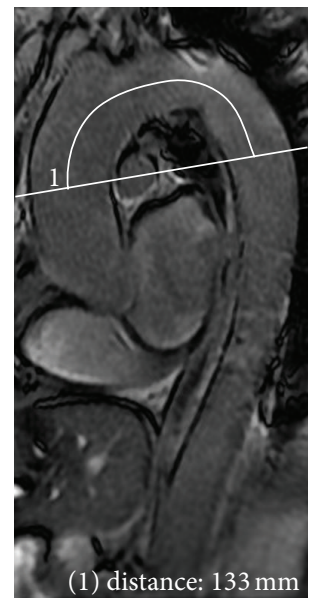

(a)

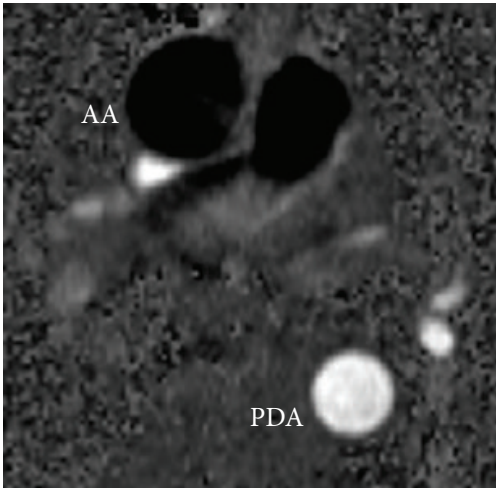

(b)

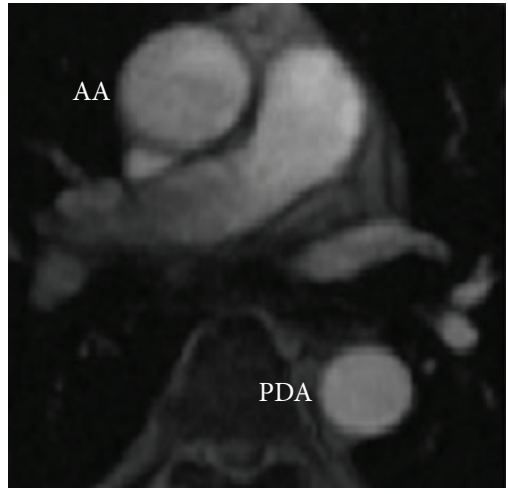

(c)

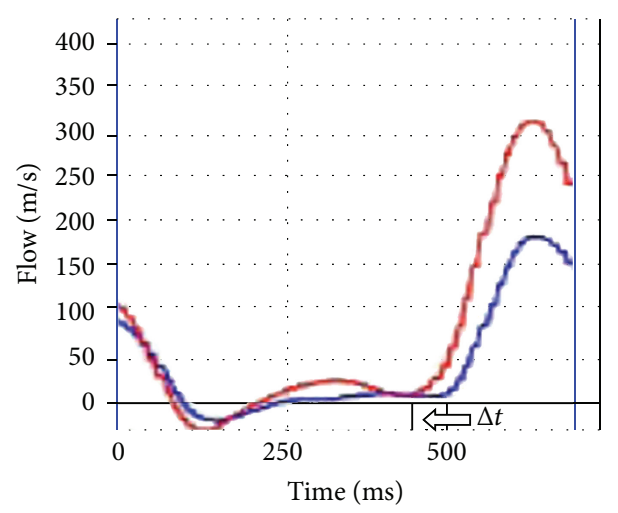

Flow 1

AA

- PDA

(d)

Figure 1: Pulse wave velocity (PWV). (a) Oblique sagittal pilot image of the aorta is used to select the plane at the level of right pulmonary artery and to measure the distance between the ascending aorta (AA) and the proximal descending aorta (PDA) by a series of short straight connected lines along the aortic luminal midline across the aortic arch at the plane. Phase (b) and magnitude (c) images acquired with an electrocardiographically gated gradient echo sequence with velocity encoding at the acquisition sites in the AA and PDA. (d) Sample flow waveforms of the ascending and proximal descending aorta over a cardiac cycle at the plane. $\Delta t$ is the time between the onsets of the systolic flow waves.

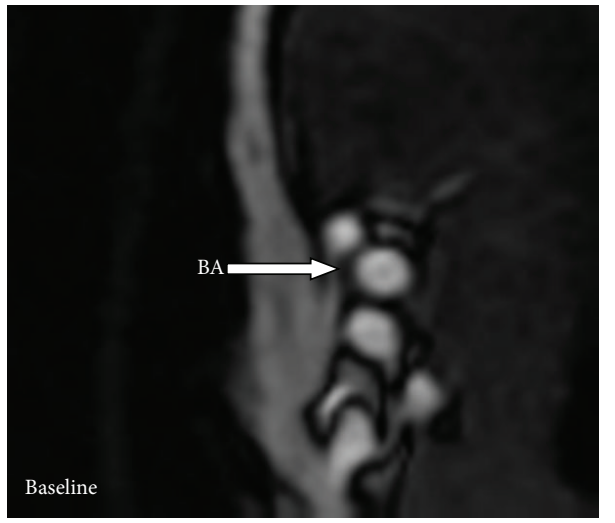

(a)

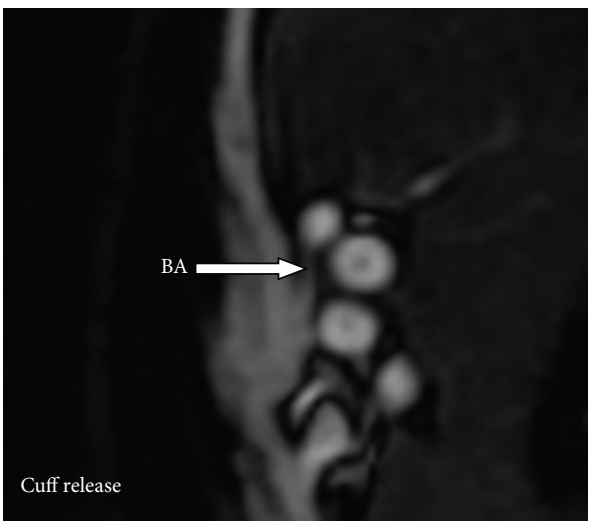

(b)

FIGURE 2: Brachial artery (BA) shown at baseline (a) and after cuff release (b). 


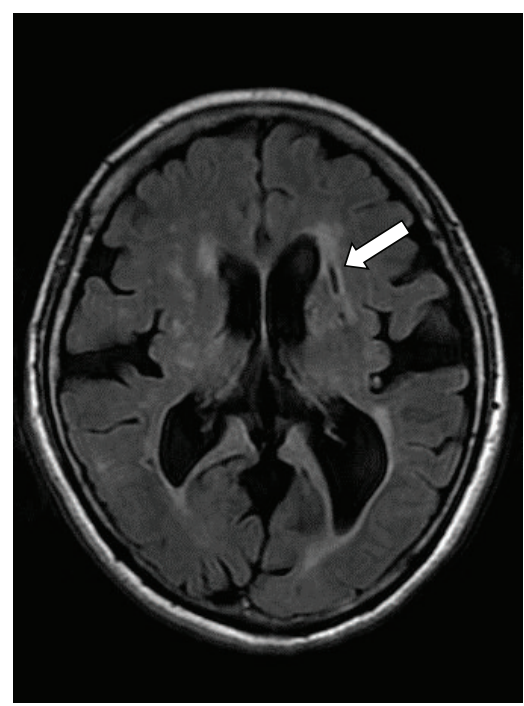

FIGURE 3: A 60-year-old female patient with type 2 diabetes mellitus for 12 years with lacunar brain infarcts (arrow) on a FLAIR sequence and aortic arch PWV of $10.56 \mathrm{~m} / \mathrm{s}$ and brachial artery FMD of $13.64 \%$.

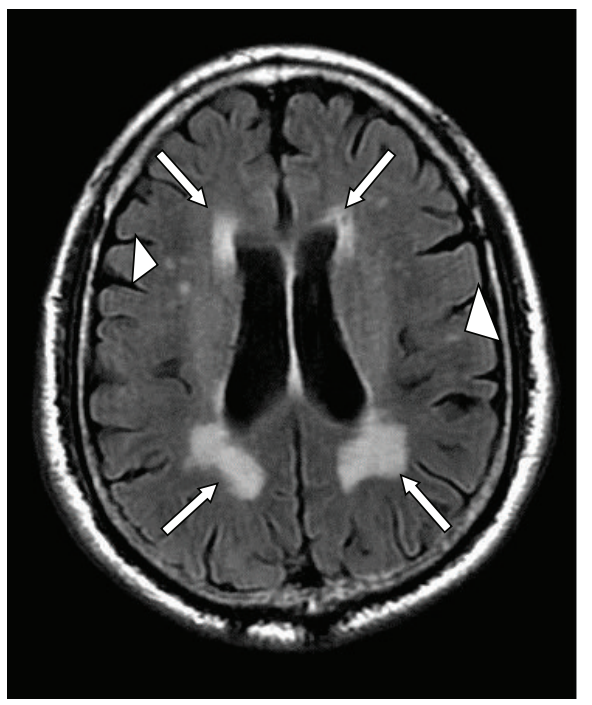

FIGURE 4: A 68-year-old male patient with type 2 diabetes mellitus for 20 years with abnormal periventricular white matter hyperintensities (WMHs) (arrows) and deep WMHs (arrowheads) on a FLAIR sequence and aortic arch PWV of $7.44 \mathrm{~m} / \mathrm{s}$ and brachial artery FMD of $5.88 \%$.

of brain parenchyma with increased signal on T2-weighted and FLAIR images without mass effect [19]. WMHs were distinguished as either periventricular (pv) WMHs or deep WMHs because of the different pathogenesis involved [18]. WMHs were classified according to Fazekas et al. Fazekas scores of 0 and 1 were considered normal, a score of 2 was considered abnormal below the age of 75 years, and a score of 3 was considered abnormal in any age group [19]. WMHs and lacunar infarcts were visually scored by a consensus reading by two neuroradiologists (10 years of experience in neuroradiology).
TABLE 2: Clinical and biochemical characteristics of the study population.

\begin{tabular}{lc}
\hline & $\begin{array}{c}\text { DM2 patients } \\
(n=62)\end{array}$ \\
\hline Sex & 37 \\
$\quad$ Male & 25 \\
$\quad$ Female & $56.84 \pm 7.46$ \\
Age (years) & $7.29 \pm 5.92$ \\
Diabetes duration (years) & $9.65 \pm 2.97$ \\
HbAlc (\%) & $133.06 \pm 16.00$ \\
Systolic blood pressure (mmHg) & $85.95 \pm 10.84$ \\
Diastolic blood pressure (mmHg) & $47.11 \pm 9.73$ \\
Pulse pressure (mmHg) & $70.48 \pm 10.49$ \\
Heart rate (bpm) & $24.92 \pm 3.95$ \\
Body mass index (kg/m $\left.{ }^{2}\right)$ & \\
Smoking & 46 \\
$\quad$ Yes & 16 \\
$\quad$ No & \\
Hypertension & 31 \\
Yes & 31 \\
$\quad$ No & $4.87 \pm 1.17$ \\
Cholesterol (mmol/L) & $1.17 \pm 0.31$ \\
HDL (mmol/L) & $2.69 \pm 1.01$ \\
LDL (mmol/L) & $2.36 \pm 2.40$ \\
Triglycerides (mmol/L) & \\
\hline
\end{tabular}

Note: values are mean \pm SD or data are numbers of patients, DM2: type 2 diabetes mellitus patients, HbAlc: glycated hemoglobin AlC, HDL: high-density lipoprotein, and LDL: low-density lipoprotein. Normal range: systolic/diastolic blood pressure $<140 / 90 \mathrm{mmHg}$, heart rate $(60-$ $100 \mathrm{bpm}), \mathrm{HbAlc}(4.0-6.0 \%)$, cholesterol $(<5.2 \mathrm{mmol} / \mathrm{L})$, triglycerides $(0.6-$ $1.7 \mathrm{mmol} / \mathrm{L}), \mathrm{HDL}(>1.04 \mathrm{mmol} / \mathrm{L})$, and LDL $(<3.12 \mathrm{mmol} / \mathrm{L})$.

2.3. Statistical Analyses. All data analysis was performed by an experienced radiologist blinded to the examinees' clinical information. Statistical analyses were carried out using SPSS for windows (version 16.0 SPSS, Chicago, Ill). Data were expressed as mean \pm standard deviation unless stated otherwise. Pearson correlation analyses were performed to analyze the association between measured parameters (aortic arch PWV, brachial FMD) and continuous data. Spearman correlation analyses were performed to analyze the association between measured parameters (aortic arch PWV and brachial FMD) and categorical data. Pearson correlation coefficients $(r)$, Spearman correlation coefficients $\left(r_{s}\right)$, and $P$ values were reported. Univariable logistic regression analyses were performed to analyze the association between MRImeasured vascular indices (PWV over aortic arch and FMD) and dichotomous data. Odds ratios (ORs), 95\% confidence intervals (CIs), and $P$ values were reported. Multiple logistic regression analyses were performed to identify variables that were independently associated with cerebral indices and to adjust for confounders including age, sex, diabetes duration, hypertension, and smoking status.

\section{Results}

3.1. Clinical Characteristics of Patients. Table 2 showed the clinical and biochemical characteristics of the study population. 
TABLE 3: Association between aortic arch PWV and cerebral lesions.

\begin{tabular}{lccc}
\hline Parameter & Number of patients & PWV & OR \\
Lacunar brain infarcts & 9 & 1.65 & $1.08-2.53$ \\
$\quad$ Yes & 53 & & $<0.05$ \\
No & & 1.58 & $1.10-2.27$ \\
Periventricular WMHs & 18 & & $<0.05$ \\
$\quad$ Yes & 44 & $1.07-2.17$ & $<0.05$ \\
No & & & \\
Deep WMHs & 28 & & \\
Yes & 34 & & \\
No & &
\end{tabular}

TABLE 4: Association between brachial artery FMD and cerebral lesions.

\begin{tabular}{lccc}
\hline Parameter & Number of patients & OR & FMD \\
Lacunar brain infarcts & 9 & & 0.22 \\
$\quad$ Yes & 53 & & \\
No & & 0.88 & $0.80-0.97$ \\
Periventricular WMHs & 18 & & $<0.01$ \\
$\quad$ Yes & 44 & 0.91 & $0.85-0.98$ \\
No & & & $<0.05$ \\
Deep WMHs & 28 & & \\
Yes & 34 & & \\
No & & \\
\hline
\end{tabular}

3.2. $P W V$ and FMD. Mean PWV over the aortic arch was $6.73 \pm 2.00 \mathrm{~m} / \mathrm{s}$ in DM2 patients. There was a significant correlation between aortic arch PWV and age $(r=$ 0.305, $P<0.05)$ and between PWV and hypertension $\left(r_{s}=\right.$ $0.498, P<0.001)$. Sex, smoking status, glycated hemoglobin A1C (HbAlc), heart rate, body mass index, lipid status, and diabetes duration did not correlate with aortic arch PWV.

Mean brachial artery FMD was $16.67 \pm 9.11 \%$. FMD was significantly associated with age $(r=-0.383, P<0.01)$ and hypertension $\left(r_{s}=-0.46, P<0.001\right)$. FMD was not found associated with sex, smoking status, glycated hemoglobin $\mathrm{A} 1 \mathrm{C}$ (HbAlc), heart rate, body mass index, lipid status, and diabetes duration.

\subsection{Association of Aortic Compliance and Brachial Endothelial} Function with Cerebral Lesions. Among 62 DM2 patients, lacunar brain infarcts were diagnosed in 9 patients. Fazekas grade 2 or 3 periventricular WMHs were diagnosed in 18 patients. Fazekas grade 2 or 3 deep WMHs were diagnosed in 28 patients.

Univariable logistic regression analyses showed that aortic arch PWV was associated with lacunar brain infarcts, Fazekas grade 2 or 3 periventricular WMHs, and Fazekas grade 2 or 3 deep WMHs (Table 3 ). Brachial artery FMD was associated with Fazekas grade 2 or 3 periventricular WMHs and Fazekas grade 2 or 3 deep WMHs (Table 4).

After adjustment for age, sex, smoking status, diabetes duration, and hypertension, aortic arch PWV was significantly associated with lacunar brain infarcts $(O R=2.00 ; 95 \%$
CI: $1.14-3.2 ; P<0.05)$, but not with periventricular and deep WMHs. Brachial artery FMD was associated with Fazekas grade 2 or 3 periventricular WMHs (OR $=0.82$; 95\% CI: $0.71-$ 0.95; $P<0.05)$.

\section{Discussion}

4.1. Association between Aortic Compliance and Cerebral Lesions. Applanation tonometry is a well recognized technique for estimating pressure waveforms and arterial stiffness [20]. However, concerning aortic stiffness, this technique can only provide an estimation of PWV along the whole carotid-femoral artery path. Furthermore, tonometry uses body surface measurements to approximate artery length and does not take into account the often torturous route of the vessels. MRI is increasingly used for measuring aortic arch PWV by using accurate aortic length and transit time between flow waves $[21,22]$. With the application of threedimensional (3D) imaging approaches, more parameters including PWV can be measured by MRI and the estimation of arterial stiffness should be accurate and comprehensive [23]. Therefore, PWV measured by MRI was used in our study.

PWV adopted by ESH guidelines is cfPWV (carotidfemoral pulse wave velocity) [24], which is different from that measured by MRI in our study. However, PWV in type 2 diabetes mellitus in our study is higher than that in healthy volunteers measured by the same MR technique [13]. The results are also consistent with the previous report 
[5]. Therefore, we think that PWV measured by MRI in our study is reliable and can reflect worse diabetic state (HbAlc $\sim 9 \%$ ) that could suggest target organ damage in the study population. In addition, the techniques used to assess arterial stiffness may not be interchangeable in clinical and research settings and comparisons of findings obtained with different arterial stiffness measures should be conducted with caution [25].

After statistical adjustment for confounding factors of age, sex, smoking status, diabetes duration, and hypertension, our study reveals that aortic arch PWV was significantly associated with lacunar brain infarcts in DM2 patients. Previous studies have shown that DM2 is a powerful risk factor of cardiovascular complications and can make aortic compliance decrease with higher $\operatorname{PWV}[5,26]$. The association between aortic stiffness and cerebral damage may be indirect with some common vascular risk factors or may be causative in nature [7]. One possible mechanism is that increased aortic stiffness leads to a deficient absorption of the pulse wave and an increase in central pulse pressure, which may augment small vessel disease of the brain through high pulsatile flow. And high pulse pressure blood flow may lead to the brain microvascular damage, including damage to vascular endothelial cells and smooth muscle cells. These situations may cause blood supply to small brain vessels to decrease or interrupt, resulting in cerebral small vessel disease and final strokes [27]. Our results suggest this assumed pathophysiologic mechanism behind the association between aortic stiffness and lacunar brain infarcts in patients with DM2.

Although there are certain common underlying risk factors causing the occurrence of WMHs and cerebral lacunar infarction $[28,29]$, we found no significant association between aortic arch PWV and WMHs after correction for confounding factors. The possible reason is that the perforating arteries may be affected more than the medullary arteries by reduced aortic compliance which causes the arteriosclerosis in the brain. Damage to the penetrating artery is mostly responsible for lacunar cerebral infarction while medullary artery damage causes WMHs.

\subsection{Association between Endothelial Function and Cere-} bral Lesions. Endothelial dysfunction is a systemic disorder, which is characterized by a reduction of the bioavailability of vasodilators, in particular, nitric oxide (NO), and increase of endothelium-derived contracting factors [30]. This imbalance leads to an impairment of the endothelium-dependent vasodilatation, which represents the functional characteristic of endothelial dysfunction. Endothelial dysfunction, which is predisposing to thrombosis, leukocyte adhesion, and smooth muscle cell proliferation, plays a pivotal role in the development, progression, and clinical manifestations of atherosclerosis [31]. Endothelial dysfunction is thought to be one of the important causes of cerebral small vessel disease and an independent predictor of stroke $[32,33]$. It has been shown that chronic endothelial dysfunction plays an important role in ischemic leukoaraiosis in cerebral small vessel disease and is related to early deterioration of brain function and poor prognosis of stroke [34]. In addition, vessel injury may cause serious consequences after occurrence of acute ischemic stroke in patients with endothelial dysfunction, due to their decreased vessel protection [8]. WMHs are associated with accelerated stroke and death [35]. Our research showed a significant correlation between FMD and pvWMHs. Therefore, by measuring the brachial artery FMD, we can potentially stratify the risks in DM2 patients. However, no association was found between FMD and deep WMHs and cerebral lacunar infarction. This difference might be attributed to different pathogenic mechanisms behind lacunar infarction, pvWMHs, and deep WMHs.

Our study had some limitations. First, this was a crosssectional study, and therefore causative mechanisms of cerebral small vessel disease cannot be determined. Furthermore, no age-matched healthy subjects were included to serve as controls. Although renal dysfunction as a risk factor for PWV has been reported in patients with type $1 \mathrm{DM}$ [36], there are no nontraditional risk factors, such as renal function and anemia in our study, which may be due to the small size of sample. However, the primary purpose of this study was to assess the possible relationship between cerebral endorgan damage and aortic compliance and brachial endothelial function in DM2 patients.

In conclusion, aortic arch PWV and brachial FMD measured with high-resolution MRI may be potentially useful to stratify DM2 patients with risk of cerebral small vessel disease.

\section{Competing Interests}

The authors declare that they have no competing interests.

\section{Acknowledgments}

This work was supported by Key Basic Research Project of Shanghai Science and Technology (no. 08JC1404500), grant from Shanghai Health and Family Planning Committee (no. XBR2013115), and Outstanding Youth Program from Zhongshan Hospital (2015ZSYXQN02).

\section{References}

[1] A. Tuttolomondo, D. Di Raimondo, R. Di Sciacca et al., "Arterial stiffness and ischemic stroke in subjects with and without metabolic syndrome," Atherosclerosis, vol. 225, no. 1, pp. 216219, 2012.

[2] S. L. Norris, D. Kansagara, C. Bougatsos, and R. Fu, "Screening adults for type 2 diabetes: a review of the evidence for the U.S. Preventive Services Task Force," Annals of Internal Medicine, vol. 148, no. 11, pp. 855-868, 2008.

[3] K. K. Naka, K. Papathanassiou, A. Bechlioulis et al., "Determinants of vascular function in patients with type 2 diabetes," Cardiovascular Diabetology, vol. 11, no. 1, article 127, 2012.

[4] G. Arcaro, A. Cretti, S. Balzano et al., "Insulin causes endothelial dysfunction in humans: sites and mechanisms," Circulation, vol. 105, no. 5, pp. 576-582, 2002.

[5] R. W. van der Meer, M. Diamant, J. J. M. Westenberg et al., "Magnetic resonance assessment of aortic pulse wave velocity, aortic distensibility, and cardiac function in uncomplicated 
type 2 diabetes mellitus," Journal of Cardiovascular Magnetic Resonance, vol. 9, no. 4, pp. 645-651, 2007.

[6] C. Vlachopoulos, K. Aznaouridis, and C. Stefanadis, "Prediction of cardiovascular events and all-cause mortality with arterial stiffness: a systematic review and meta-analysis," Journal of the American College of Cardiology, vol. 55, no. 13, pp. 1318$1327,2010$.

[7] S. Laurent, S. Katsahian, C. Fassot et al., "Aortic stiffness is an independent predictor of fatal stroke in essential hypertension," Stroke, vol. 34, no. 5, pp. 1203-1206, 2003.

[8] A. M. Tiehuis, Y. van der Graaf, F. L. Visseren et al., "Diabetes increases atrophy and vascular lesions on brain MRI in patients with symptomatic arterial disease," Stroke, vol. 39, no. 5, pp. 1600-1603, 2008.

[9] J. Stamler, O. Vaccaro, J. D. Neaton, and D. Wentworth, "Diabetes, other risk factors, and 12-yr cardiovascular mortality for men screened in the multiple risk factor intervention trial," Diabetes Care, vol. 16, no. 2, pp. 434-444, 1993.

[10] S. G. C. van Elderen, A. Brandts, J. J. M. Westenberg et al., "Aortic stiffness is associated with cardiac function and cerebral small vessel disease in patients with type 1 diabetes mellitus: assessment by magnetic resonance imaging," European Radiology, vol. 20, no. 5, pp. 1132-1138, 2010.

[11] D. Santos-García, M. Blanco, J. Serena et al., "Brachial arterial flow mediated dilation in acute ischemic stroke," European Journal of Neurology, vol. 16, no. 6, pp. 684-690, 2009.

[12] Y. Shan, J. Lin, P. Xu, M. Zeng, H. Lin, and H. Yan, “The combined effect of hypertension and type 2 diabetes mellitus on aortic stiffness and endothelial dysfunction: an integrated study with high-resolution MRI," Magnetic Resonance Imaging, vol. 32, no. 3, pp. 211-216, 2014.

[13] Y. Shan, J. Lin, P. Xu, J. Zhou, and M. Zeng, "Comprehensive assessment of aortic compliance and brachial endothelial function using 3.0-T high-resolution MRI: a feasibility study," Journal of Computer Assisted Tomography, vol. 36, no. 4, pp. 437442, 2012.

[14] H. Bokura, S. Kobayashi, and S. Yamaguchi, "Distinguishing silent lacunar infarction from enlarged Virchow-Robin spaces: a magnetic resonance imaging and pathological study," Journal of Neurology, vol. 245, no. 2, pp. 116-122, 1998.

[15] K. G. Alberti and P. Z. Zimmet, "Definition, diagnosis and classification of diabetes mellitus and its complications-part 1: diagnosis and classification of diabetes mellitus. Provisional report of a WHO consultation," Diabetic Medicine, vol. 15, no. 7, pp. 539-553, 1998.

[16] S. M. Grundy, J. I. Cleeman, S. R. Daniels et al., "Diagnosis and management of the metabolic syndrome: an American Heart Association/National Heart, Lung, and Blood Institute Scientific Statement," Circulation, vol. 112, no. 17, pp. 2735-2752, 2005.

[17] S. E. Vermeer, W. T. Longstreth Jr., and P. J. Koudstaal, "Silent brain infarcts: a systematic review," The Lancet Neurology, vol. 6, no. 7, pp. 611-619, 2007.

[18] B. H. Braffman, R. A. Zimmerman, J. Q. Trojanowski et al., "Brain MR: pathologic correlation with gross and histopathology. I. Lacunar infarction and Virchow-Robin spaces," American Journal of Roentgenology, vol. 151, pp. 551-558, 1988.

[19] F. Fazekas, R. Schmidt, and P. Scheltens, "Pathophysiologic mechanisms in the development of age-related white matter changes of the brain," Dementia and Geriatric Cognitive Disorders, vol. 9, supplement 1, pp. 2-5, 1998.
[20] S. Laurent, J. Cockcroft, L. Van Bortel et al., "Expert consensus document on arterial stiffness: methodological issues and clinical applications," European Heart Journal, vol. 27, no. 21, pp. 2588-2605, 2006.

[21] H. B. Grotenhuis, J. J. M. Westenberg, P. Steendijk et al., "Validation and reproducibility of aortic pulse wave velocity as assessed with velocity-encoded MRI," Journal of Magnetic Resonance Imaging, vol. 30, no. 3, pp. 521-526, 2009.

[22] E.-S. Ibrahim, K. Johnson, A. Miller, J. Shaffer, and R. White, "Measuring aortic pulse wave velocity using high-field cardiovascular magnetic resonance: comparison of techniques," Journal of Cardiovascular Magnetic Resonance, vol. 12, article 26, 2010.

[23] P. Dyverfeldt, T. Ebbers, and T. Länne, "Pulse wave velocity with 4D flow MRI: systematic differences and age-related regional vascular stiffness," Magnetic Resonance Imaging, vol. 32, no. 10, pp. 1266-1271, 2014.

[24] G. Mancia, R. Fagard, K. Narkiewicz et al., "ESH/ESC guidelines for the management of arterial hypertension: the Task Force for the Management of Arterial Hypertension of the European Society of Hypertension (ESH) and of the European Society of Cardiology (ESC)," European Heart Journal, vol. 34, pp. 21592219, 2013.

[25] J. Lim, M. Pearman, W. Park, M. Alkatan, and H. Tanaka, "Interrelationships among various measures of central artery stiffness," American Journal of Hypertension, 2016.

[26] J. M. S. Lee, C. Shirodaria, C. E. Jackson et al., "Multi-modal magnetic resonance imaging quantifies atherosclerosis and vascular dysfunction in patients with type 2 diabetes mellitus," Diabetes and Vascular Disease Research, vol. 4, no. 1, pp. 44-48, 2007.

[27] D.-H. Kim, J. Kim, J.-M. Kim, and A. Y. Lee, "Increased brachialankle pulse wave velocity is independently associated with risk of cerebral ischemic small vessel disease in elderly hypertensive patients," Clinical Neurology and Neurosurgery, vol. 110, no. 6, pp. 599-604, 2008.

[28] M. F. O'Rourke and M. E. Safar, "Relationship between aortic stiffening and microvascular disease in brain and kidney: cause and logic of therapy," Hypertension, vol. 46, no. 1, pp. 200-204, 2005.

[29] N. Altaf, P. S. Morgan, A. Moody, S. T. MacSweeney, J. R. Gladman, and D. P. Auer, "Brain white matter hyperintensities are associated with carotid intraplaque hemorrhage," Radiology, vol. 248, no. 1, pp. 202-209, 2008.

[30] A. Lerman and J. C. Burnett Jr., "Intact and altered endothelium in regulation of vasomotion," Circulation, vol. 86, no. 6, supplement, pp. III12-III19, 1992.

[31] R. Ross, "Atherosclerosis-an inflammatory disease," The New England Journal of Medicine, vol. 340, no. 2, pp. 115-126, 1999.

[32] J. Yeboah, J. R. Crouse, F.-C. Hsu, G. L. Burke, and D. M. Herrington, "Brachial flow-mediated dilation predicts incident cardiovascular events in older adults: the cardiovascular health study," Circulation, vol. 115, no. 18, pp. 2390-2397, 2007.

[33] N. Gokce, J. F. Keaney Jr., L. M. Hunter et al., "Predictive value of noninvasively determined endothelial dysfunction for longterm cardiovascular events in patients with peripheral vascular disease," Journal of the American College of Cardiology, vol. 41, no. 10, pp. 1769-1775, 2003.

[34] A. Hassan, B. J. Hunt, M. O’Sullivan et al., "Markers of endothelial dysfunction in lacunar infarction and ischaemic leukoaraiosis," Brain, vol. 126, no. 2, pp. 424-432, 2003. 
[35] K. S. King, K. X. Chen, K. M. Hulsey et al., "White matter hyperintensities: use of aortic arch pulse wave velocity to predict volume independent of other cardiovascular risk factors," Radiology, vol. 267, no. 3, pp. 709-717, 2013.

[36] S. G. C. van Elderen, J. J. M. Westenberg, A. Brandts et al., "Increased aortic stiffness measured by MRI in patients with type 1 diabetes mellitus and relationship to renal function," American Journal of Roentgenology, vol. 196, no. 3, pp. 697-701, 2011. 


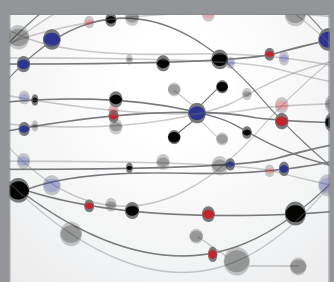

The Scientific World Journal
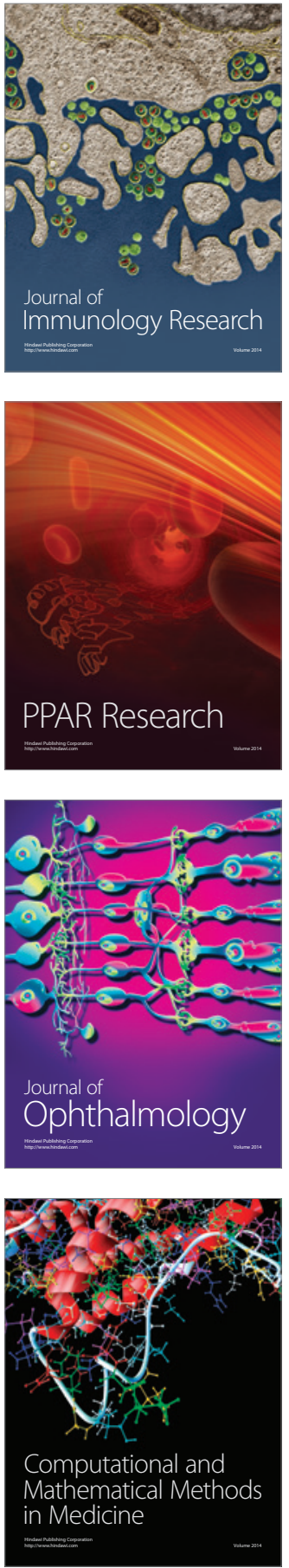

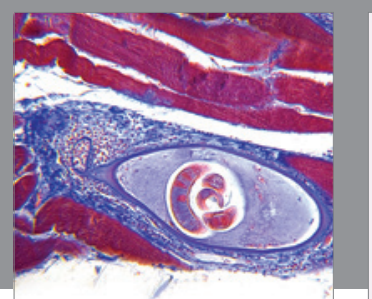

Gastroenterology Research and Practice

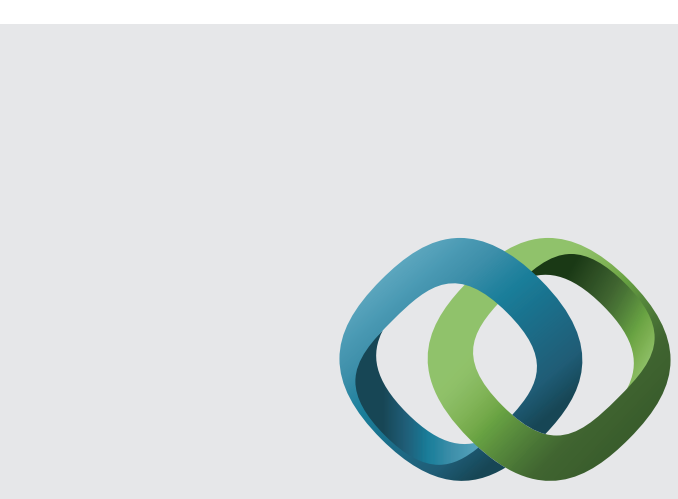

\section{Hindawi}

Submit your manuscripts at

http://www.hindawi.com
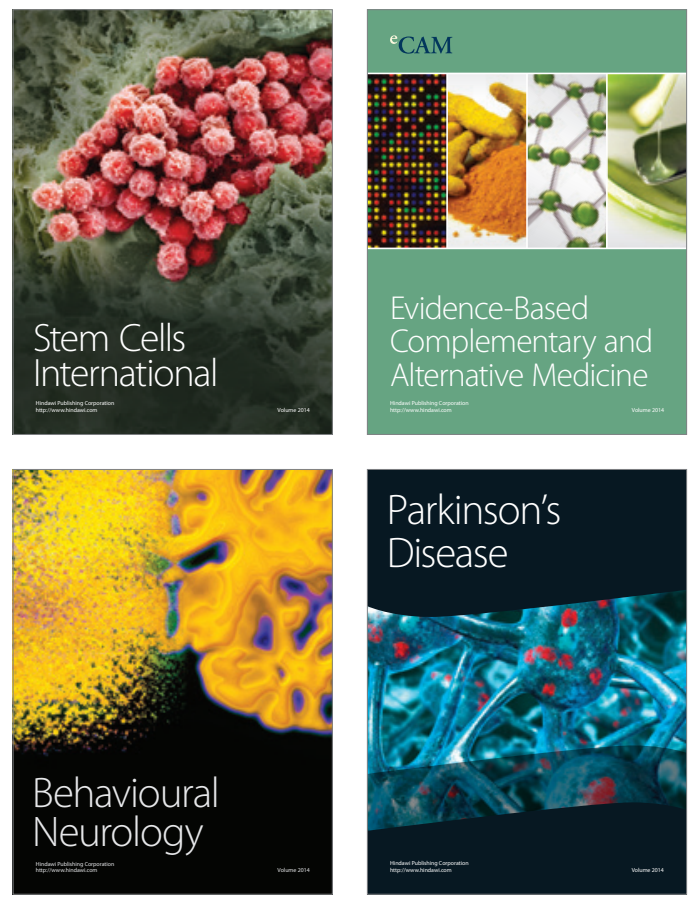
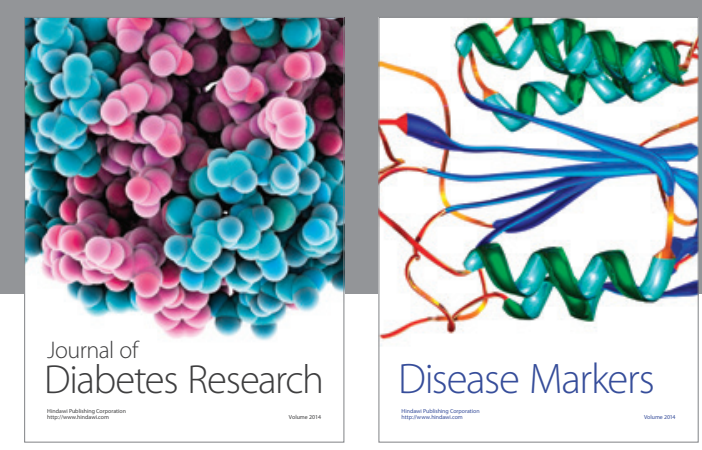

Disease Markers
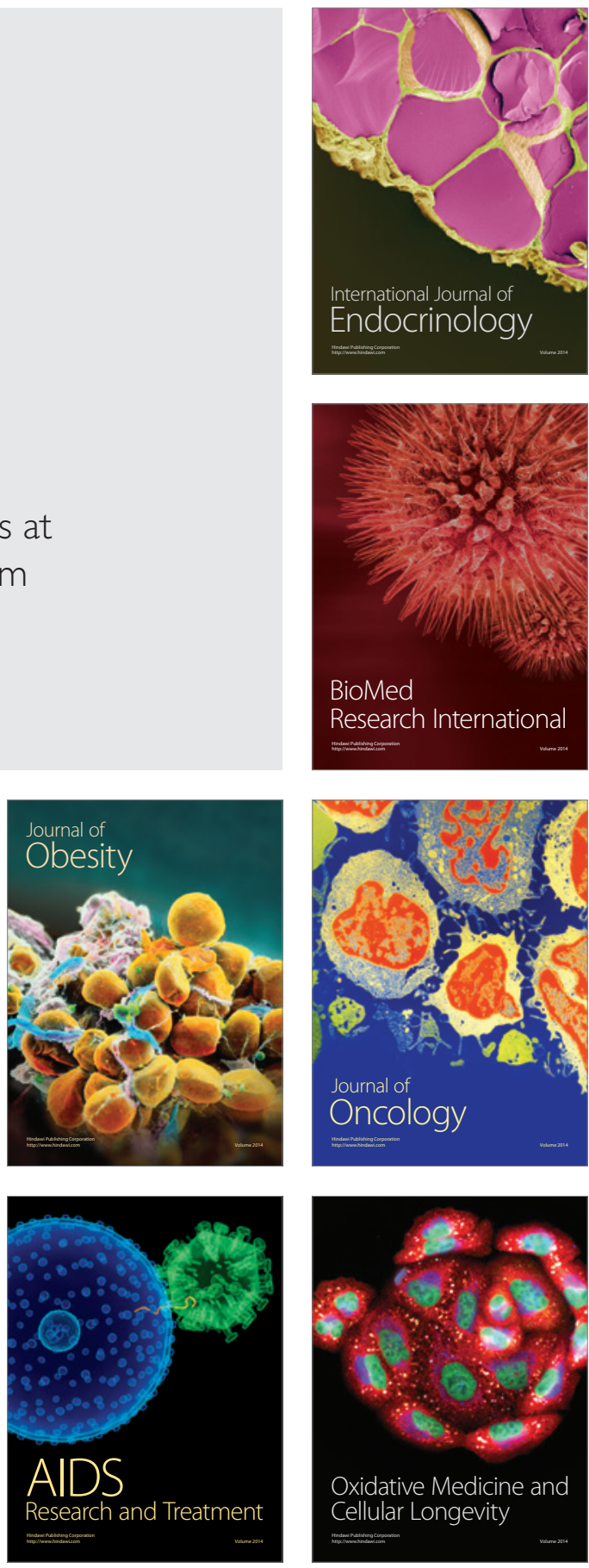